\title{
CUMULATIVE AUTHOR INDEX
}

\section{Abad, J. and \\ R. Rodriguez-Trias}

Abdalla, E., M. C. B.

Abdalla and A. Lima

Santos

Abdalla, M.C.B.

Abecasis, C., A. Foussats

and $\mathrm{O}$. Zandron

Amati, D., M. Ciafaloni and $\mathrm{G}$. Veneziano

Ametller, L1. and

D. Treleani

Anand, Dev

Angus, Ian G.

Anishetty, R.,

Rahul Basu and

H. S. Sharatchandra

Anisovich, V. V., M. N. Kobrinsky, V. A. Nikonov and

J. Nyiri

Arash, Firooz, Michael

J. Moravcsik and Gary R. Goldstein

Aratyn, $H$. and

A. H. Zimerman

Arnowitt, R., V. Barger

M. Barnett, R. Blair,

L. Clavelli,

N. G. Deshpande,

M. Drees, K. Enqvist,

J. Fisher, R. Godbole,

J. Gunion, H. Haber,

K. Hagiwara,
Study of the BRS Charge in the Polyakov String by the Kugo-Ojima Method

A Nonlinear $\sigma$-Model Related to the Fine Structure of Strings

see Abdalla, E.

Diffeomorphically Nonequivalent

Classes of Solutions in Gauge

Theories on Group Manifolds

Classical And Quantum Gravity Effects

from Planckian Energy Superstring

Collisions

Shadowing in Semihard Interactions

see Banerjee, S.

Indications of $A \Delta I=1 \backslash 2$ Rule in the

Strong Coupling Regime

A General' Property of Spontaneously

Broken SUSY Theories

$1 / N_{c}$ Expansion, Quark Model and

Hadron Collisions at High Energies

Simple Reaction Structures in a

"Complicated" Kinematic Range

Ghosts and the Physical Modes in the

Covariant Free String Field Theories

Superstrings: Group Report

A3 (1988) 1615

A3 (1988) 521

A3 (1988) 825

A3 (1988) 1385

A3 (1988) 875

A1 (1986) 463

A2 (1987) 739

A1 (1986) 421

A2 (1987) 1097 
M. Herrero, J. Hewett,

K. Igi, D. Karatas,

C. S. Kim, E. Ma,

A. Mendez, S. Nandi,

P. Nath, T. Rizzo,

N. Tracas, X. Tata and

$\mathrm{K}$. Whisnant

Arnowitt, R.,

Weak Gaugino Production at the SSC

A2 (1987) 1113

R. M. Barnett, Pran

Nath and F. Paige

Atre, M. V.,

A. P. Balachandran

and T. R. Govindarajan

Atwood, D.

Avan J. and

H. J. de Vega

Massless Spinning Particles in all

Dimensions and Novel Magnetic

A2 (1987) 453

Monopoles

see Contogouris, A. P.

Conformally Covariant Approach to

A2 (1987) 1075

the Integrability of SDYM: Linear

System, $\beta$-planes Infinitesimal

Backlund Transformations and

Infinite Dimensional Algebras

Aziz, T.

A New Scaling in the Rapidity Dis-

tribution of Particles Produced in

High Energy Hadronic Collisions

A3 (1988) 1263

A1 (1986) 991

Baba, P. V. K. S.

see Banerjee, $\mathrm{S}$.

A3 (1988) 825

Babu, P. Ram

Testing Some Properties of Neutral

A3 (1988) 721

Current Neutrino Interactions in

Neutrino Counting Reaction

Baer, $\mathbf{H}$.

see Gunion, J. F.

A2 (1987) 1145

Gluino Decays to $W$ and $Z$ Bosons at the SSC

Baer, Howard,

R. Michael Barnett

Manuel Drees,

John F. Gunion,

Howard E. Haber,

Debra L. Karatas and

Xerxes R. Tata

Bagneid, A.

see Barger, V.

Bagneid, A., T. K. Kuo and N. Nakagawa

Baker, W. F.

Balachandran, A. P.

Balachandran, A. P.,

B. Rai, G. Sparano and

A. M. Srivastava

Bandō, H., Y. Shono and

H. Takaki

Banerjee, S.,

Unification of Electroweak and

A2 (1987) 1327

Horizontal Interactions

A2 (1987) 1351

Small $t$ Physics at the Tevatron Collider

A2 (1987) 891

see Atre, M. V.

A2 (1987) 453

Theory and Phenomenology of Isospin

A3 (1988) 2621

Balls

$\Lambda-\Sigma$ Coupling Effect on Nonmesonic

A3 (1988) 1581

Decay of Hypernuclei

Triple Regge Analysis and $K \bar{K}$ Off-

A3 (1988) 825 
S. N. Ganguli,

A. Gurtu,

P. K. Malhotra,

K. Sudhakar,

Y. Goldschmidt-

Clermont, R. T. Ross,

S. Squarcia, T. Haupt, M. W. Krasny,

P. Girtler, D. Kuhn,

G. L. Kaul, Dev Anand,

P. V. K. S. Baba,

K. W. J. Barnham,

R. M. Eason, F. Mandl,

M. Markytan,

M. Bardadin-

Otwinowska and

M. Szczekowski

Banerjee, S. N.,

R. K. Das and

A. K. Sarker

Baranov, M. A. and

A. S. Schwarz

Barclay, M.

Bardadin-

Otwinowska, M.

Barger, V.

Barger, V. and

K. Whisnant

Barger, V.,

N. G. Deshpande and

K. Hagiwara

Barger, V.,

N. G. Deshpande,

T. Kuo, A. Bagneid,

S. Pakvasa and

K. Whisnant

Barger, V. and

K. Whisnant

Barger, V. and

K. Whisnant

Barger, V., J. L. Lopez and W. Putikka
Shell Interactions from $p \stackrel{K^{-}}{\rightarrow} \Lambda$ at

$110 \mathrm{GeV} / \mathrm{c}$

On the Structure of $\pi$ and $K$ Mesons

A2 (1987) 1829

On the Multiloop Contribution to the

A2 (1987) 1773

String Theory

see Ramayya, A. V.

A3 (1988) 499

see Banerjee, $\mathrm{S}$.

A3 (1988) 825

see Arnowitt, R.

Heavy $Z$ Boson Decays to $W, Z$ and

A2 (1987) 1097

A2 (1987) 1171

Higgs Bosons in $E_{6}$ Superstring

Models

Signal for Superstring Quark Singlet

Production: Violation of Flavor

Universality

Discovery Limits of New Gauge

Bosons of $\mathrm{S}_{\mathrm{PL}}(6) \times \mathrm{U}(1)$

A2 (1987) 1181

A2 (1987) 1327

Production and Decays of Extra Gauge

A3 (1988) 879

Bosons in a Left-Right $E_{6}$ Superstring

Model

Higgs Boson Production via $Z, W$

Bosons and Toponium in the $E_{6}$

Superstring Model

Next-to-Leading Logarithm QCD

Corrections for $q \bar{q} \rightarrow Z Z, W^{+} W^{--}$, $W^{ \pm} Z$ Subprocesses 
3014 Cumulative Author Index

Barnett, $\mathbf{M}$.

Barnett, R. M.

Barnett, R. M.

Barnett, R. Michael

Barnham, K. W. J.

Barut, A. O. and

R. Raczka

Basu, Rahul

Baur, U., I. Hinchliffe

and D. Zeppenfeld

Beilin, V. A.,

V. A. Nesterenko and

A. V. Radyushkin

Bengtsson, H.-U.,

C. D. Buchanan and

A. Soni

Bengtsson, H.-U. and

E. W. N. Glover

Bengtsson, H.-U.,

T. D. Gottschalk and

F. E. Paige

Bengtsson, Hans-Uno, Hiroaki Yamamoto and Sachio Komamiya

Berezin, V. A.

Bershadski, M.

Bershadsky, M. and

A. Radul

Biedenharn, L. C.,

H. van Dam,

G. Marmo,

G. Morandi,

N. Mukunda,

J. Samuel and

E. C. G. Sudarshan

Bigi, I. I. and L. Clavelli

Blair, R.

Blair, R. E.

Bohm, A., L. J. Boya,

P. Kielanowski,

M. Kmiecik, M. Loewe

and P. Magnollay see Arnowitt, R.

A2 (1987) 1097

see Arnowitt, $\mathbf{R}$.

A2 (1987) 1113

see Gunion, J. F.

A2 (1987) 1145

A2 (1987) 1131

A3 (1988) 825

see Banerjee, $\mathbf{S}$

On the Strong Van Der Waals Forces

Between Hadrons Due to Confining

Potentials

see Anishetty, R.

A2 (1987) 265

A3 (1988) 875

Excited Quark Production at Hadron

Colliders

Kaon Form Factor for Small $Q^{2}$

A3 (1988) 1183

A Possible (?) Single-Muon Trigger for $B$-Physics at the SSC

Signatures and Mass Determination

of Heavy Top Quarks at the SSC

QCD Working Group Summary

A2 (1987) 1241

A2 (1987) 1255

A2 (1987) 1359

Search for Charged Higgs Bosons at

A2 (1987) 1055

SSC

Unusual Hydrodynamics

A2 (1987) 1591

On Off-Shell States in Bosonic String

Theory

Conformal Field Theories with Addi-

tional $Z_{\mathrm{N}}$ Symmetry

Classical Models for Regge

Trajectories

A1 (1986) 443

A2 (1987) 165

A2 (1987) 1567

A2 (1987) 1235

Beauty Mesons With the $J / \Psi$

Trigger

see Arnowitt, $\mathbf{R}$.

A2 (1987) 1097

A2 (1987) 1437

A3 (1988) 1103
Theory of Relativistic Extended

Objects 
Bonini, M. and R. Iengo Correlation Functions and Zero Modes on Higher Genus Riemann Surfaces

Bonneau, Guy and Francois Delduc

Boutaleb-J., H. and

A. L. Marrakchi

Boya, L. J.

Braaten, Eric,

Alan R. White and

Charles R. Willcox

Brandenberger,

Robert H.

Braun, V. M.,

L. G. Dakhno and

V. A. Nikonov

Braun, V. M. and

Yu. M. Shabelski

Bressloff, P. C.,

J. G. Taylor and

A. Restuccia

Buchanan, C. D.

Buchbinder, I. L. and

S. D. Odintsov

Construction and Properties of Quasi

Ricci Flat Spaces

A Scalar-Tensor Theory for Induced

Gravity

see Bohm, A.

Infrared Anomalous Interactions as a

Signal for Electroweak Symmetry

Breaking by Color Sextet Quarks

Inflation and Cosmic Strings: Two

Mechanisms for Producing Structure

in the Universe

Elastic $p d$ Scattering at High Energies

Multiple Scattering Theory for

Inelastic Processes

A Functional Light-Cone Gauge

Construction of a Bosonic String

Compactified on a Torus

see Bengtsson, H.-U.

Effective Action in Multi-Dimensional

Supergravities and Induced Einstein
Cahill, R. T.

Campbell, B. A., J. Ellis, Superstring Models Challenged by

K. Enquist,

M. K. Gaillard and

D. V. Nanopoulos

Carimalo, C.

Carruthers, P. and

C. C. Shih

Castellani, L.

Cecotti, S., S. Ferrara and $\mathbf{M}$. Villasante

Cecotti, S., S. Ferrara,

L. Girardello,

A. Pasquinucci and

M. Porrati

Chapline, George F. Gravity

see Praschifka, J.

Rare Processes

A3 (1988) 841

A1 (1986) 997

A2 (1987) 179

A3 (1988) 1103

A1 (1986) 693

A2 (1987) 77

A3 (1988) 1301

A3 (1988) 2417

A3 (1988) 451

A2 (1987) 1241

A3 (1988) 1859

A2 (1987) 1797

A2 (1987) 831

A2 (1987) 249

A2 (1987) 1447

A3 (1988) 1435

Supergrav-A Reduce Package for

Bose-Fermi Exterior Calculus and

the Construction of Supergravity

Actions

Linear Multiplets and Super Chern-

Simons Forms in 4D-Supergravity

Matter-Coupled Supergravity With

Gauss-Bonnet Invariants: Com-

ponent Lagrangian and Super-

symmetry Breaking

Unification of Yang-Mills Theory and Superstrings 
3016 Cumulative Author Index

Chaturvedi, S.,

A. K. Kapoor and

V. Srinivasan

Chen, Min

Chen, Su-Qing

Chia, Swee-Ping

Cho, B. H.

Chou, T. T. and

Chen Ning Yang

Chou, T. T. and

Chen Ning Yang

Ciafaloni, $\mathrm{M}$.

Clavelli, L.

Clavelli, L.

Cleemann, L.

Contogouris, A. P.,

N. Mebarki and

D. Atwood

Coquereaux, Robert

Coule, David H. and

Milan B. Mijic

Cox, B. and A. Soni

Craigie, N. S.,

E. Katznelson and

S. Mahmood

Cristofano, G.,

G. Maiella, R. Musto,

F. Nicodemi and

R. Pettorino

Csikor, F., E. Lendvai

and G. Pócsik

Cudell, J. R., F. Halzen

and C. S. Kim

D'Auria, Riccardo,

Pietro Fré, Mario

Raciti and Franco

Riva
Renormalization of Stochastically

Quantized Field Theories

A Short History of Measuring the

Strong Coupling Constant $\alpha_{s}$ to

Second Order in QCD

see Ni, Guang-Jiong

An Improved One-loop Analysis of the

$\lambda \phi^{4}$ Theory at Finite Temperature

see Kwon, O. J.

Should There be KNO Scaling for $e^{+} e^{-}$ 2-Jet Events?

A Unified Physical Picture of Multiparticle Emission: Wide Multiplicity Distribution for $\bar{p} p$ and Narrow Multiplicity Distribution for $e^{+} e^{-}$ Collisions

see Amati, D.

see Arnowitt, R.

see Bigi, I. I.

see Ramayya, A. V.

Dispersion Relations Approach for

Heavy Higgs

Comments About the Geometry of

Nonlinear Sigma Models

Quantum Fluctuations and Eternal

Inflation in the $R^{2}$ Model

Heavy Flavors (Summary Report)

A Monte Carlo Study of Spontaneous

Chiral Symmetry Breaking in Lattice

Gauge Theories

Twisted Bosonic Strings and $\mathrm{O}(16) \otimes$

O(16) Heterotic Model

Effects of An Extra Neutral $E_{6}$ Gauge

Boson in $e^{+} e^{-}$Annihilation into

Hadrons

The Standard Model and Proton-

Antiproton Colliders in the ACOL/

TEV I ERA

Anomaly Free Supergravity in

$D=10:$ I) The Bianchi Identities and

The Bosonic Lagrangian
A3 (1988) 163

A1 (1986) 669

A3 (1988) 1735

A2 (1987) 713

A1 (1986) 709

A1 (1986) 415

A2 (1987) 1727

A3 (1988) 1615

A2 (1987) 1097

A2 (1987) 1235

A3 (1988) 499

A2 (1987) 1075

A2 (1987) 1763

A3 (1988) 617

A2 (1987) 1221

A1 (1986) 953

A2 (1987) 729

A3 (1988) 2959

A3 (1988) 1051

A3 (1988) 953 


Dadhich, Naresh
Daftardar, Varsha and
Naresh Dadhich
Dakhno, L. G.
Dalitz, R. H.

Das, R. K.

de Vega, H. J.

De Azeredo Campos, R. and J. M. L. Fisch

Dehin, D. and V. Hussin

Del Duca, V.,

L. Magnea and

P. van Nieuwenhuizen

Delbourgo, R., S. Twisk and G. Thompson

Delbourgo, $\mathrm{R}$.

Delduc, Francois

Deryagin, D. V.,

D. Yu Grigoriev and

V. A. Rubakov

Deshpande, N. G.

Deshpande, N. G.

Deshpande, N. G.

Dharmaratna, W. G. D.

Dolgov, A. D. and

D. P. Kirilova

Domenech, G.,

M. L. Levinas and

N. Sánchez

Donoghue, John F.,

Barry R. Holstein and

German Valencia

Drees, $M$.

Drees, $\mathbf{M}$.

Drees, $\mathrm{M}$.

Drees, Manuel

Eason, R. M.

Eberth, J.

Ecker, G. see Daftardar, Varsha

Uncertainty Principle and the Quan-

tum Fluctuations of the Light Cones

in the Static Space-Times

see Braun, V. M.

An Outline of the Life and Work of

Tony Hilton Royle Skyrme

see Banerjee, S. N.

see Avan, J.

Hamiltonian Formulation of the $N=1$

$D=10$ Supergravity Coupled to

Super Yang-Mills Theory

Constrained Superfields and Super-

symmetric Magnetic Field Systems

Equivalence of the Lagrangian and

Hamiltonian BRST Charges for the

Bosonic String in the Harmonic and

Conformal Gauges

Massive Yang-Mills Theory:

Renormalizability Versus Unitarity

Grassmann Wave Functions and

Intrinsic Spin

see Bonneau, Guy

Cold Dense Fermionic Matter in the

Electroweak Theory: Anisotropic

$W$-Boson Condensate at $B \neq L$

see Arnowitt, R.

see Barger, V.

see Barger, V.

see Goldstein, Gary R.

Nonequilibrium Decays of Light

Particles and Primodial

Nucleosynthesis

Quantum Field Theory and the

Antipodal Identification of Space

Time

Survey of Present and Future Tests of

CP Violation

see Gunion, J. F.

see Arnowitt, R.

see Gunion, J. F.

see Baer, Howard

see Banerjee, $\mathrm{S}$.

see Ramayya, A. V.

see Neufeld, $\mathrm{H}$.
A1 (1986) 731

A1 (1986) 731

A3 (1988) 1301

A3 (1988) 2719

A2 (1987) 1829

A3 (1988) 1263

A3 (1988) 2371

A3 (1988) 487

A3 (1988) 1081

A3 (1988) 435

A3 (1988) 591

A1 (1986) 997

A3 (1988) 1199

A2 (1987) 1097

A2 (1987) 1181

A2 (1987) 1327

A3 (1988) 1847

A3 (1988) 267

A3 (1988) 2567

A2 (1987) 319

A2 (1987) 1035

A2 (1987) 1097

A2 (1987) 1145

A2 (1987) 1131

A3 (1988) 825

A3 (1988) 499

A3 (1988) 603 
3018 Cumulative Author Index

Eisenberg, J. M.

Ellis, J.

Enqvist, $\mathrm{K}$.

Enqvist, $\mathrm{K}$.

Fang, L. Z. and

Z. C. Wu

Fateev, V. A. and

S. L. Lykyanov

Ferrara, S.

Ferrara, S.

Fisch, J. M. L.

Fisher, J.

Foda, Omar

Foussats, A.

Foussats, A. and

O. Zandron

Fradkin, E. S. and

M. A. Vasiliev

Frappat, L.

Fré, Pietro

Fujitsu, Akira and

Kazuhiro Kimura

Gabbiani, F.

Gaillard, M. K.

Gan, Kock Kiam and

Martin L. Perl

Ganguli, S. N.

Garbaczewski, Piotr see Kluger, Y.

see Campbell, B. A.

see Campbell, B. A.

see Arnowitt, R.

An Overview of Quantum Cosmology

The Models of Two-Dimensional Conformal Quantum Field Theory with $Z_{n}$ Symmetry

see Cecotti, $S$.

see Cecotti, S.

see De Azeredo Campos, R.

see Arnowitt, $\mathrm{R}$.

Momentum Subtraction Renormalization Techniques in Curved

Space-Time

see Abecasis, C.

Super Yang-Mills Theories Coupled to Supergravity. Tangent Bundle

to a Supergroup Manifold

Approach

Superalgebra of Higher Spins and

Auxiliary Fields

Vertex Operator Representation of $\operatorname{OSp}(M / N)^{(\mathbf{1})}$

see D'Auria, Riccardo

Vertex Operator Construction of the

Thirring Model and the Associated

Wess-Zumino-Witten Model in Two

Dimensions

A Cosmologically Acceptable Harmless Axion in a Superstring-Inspired Model

see Campbell, B. A.

New Leptons: An Experimental Review

see Banerjee, $\mathbf{S}$.

Canonical Action-Angle Formalism for

Quantized Nonlinear Fields

Gastmans, R., A. Sevrin, Infinite Dimensional Extended Super-

W. Troost and

A. Van Proeyen

Gatto, R. and G. Sartori conformal Lie Algebras

Relics of Supersymmetry in the Mass

Spectrum after Spontaneous Breaking
A3 (1988) 2127

A2 (1987) 831

A2 (1987) 831

A2 (1987) 1097

A1 (1986) 887

A3 (1988) 507

A2 (1987) 1839

A3 (1988) 1675

A3 (1988) 2371

A2 (1987) 1097

A2 (1987) 1549

A3 (1988) 2303

A3 (1988) 2971

A3 (1988) 2983

A3 (1988) 2545

A3 (1988) 953

A3 (1988) 2911

A3 (1988) 397

A2 (1987) 831

A3 (1988) 531

A3 (1988) 825

A2 (1987) 223

A2 (1987) 195

A1 (1986) 683 
see Cecotti, S.

see Banerjee, S.

see Bengtsson, H.-U.

The $\gamma \gamma$ Signal of Pseudoscalar

$t^{\prime}$-Quarkonium at the SSC

Godbole, R.

Godbole, R.

Godbole, R. M. and

F. Olness

Goddard, Peter and

David Olive

Goldschmidt-Clermont, see Banerjee, S.

Y.

Goldstein, Gary R.

Goldstein, Gary R.,

W. G. D. Dharmaratna,

Michael J. Moravcsik

and Gordon A.

Ringland

Goldstein, Gary R. and

Michael J. Moravcsik

Goncharov, A. S.,

A. D. Linde and

V. F. Mukhanov

Gottschalk, T. D.

Gottschalk, Thomas D.

Govindarajan, T. R.

Govindarajan, T. R., Anjan S. Joshipura, Saurabh D. Rindani and Utpal Sarkar

Greiner, Walter

Grifols, J. A.,

S. Peris and J. Solà

Grimus, W.

Gunion, J., A. Mendez

and F. Olness

Gunion, J.

Gunion, J. F. and

H. E. Haber

Gunion, J. F.,

H. E. Haber,

see Gunion, J. F.

see Arnowitt, R.

the Equivalent Vector Boson

Approximation

see Arash, Firooz

Polarized Particles

tionary Universe

see Bengtsson, H.-U.

Monte Carlo Shower Models

see Atre, M. V.

Gaugino Condensation

see Schäfer, Andreas

the Scale of Compositeness

see Neufeld, $H$.

Is the Left-Right Higgs Sector

Observable?

see Arnowitt, R.

(Group Summary)
Contribution of Transverse Gauge

Bosons to Higgs Production and

Kac-Moody and Virasoro Algebras in

Relation to Quantum Physics

Inclusive Reactions With Three

Inclusive Reactions and Total Cross

Sections with Polarized Initial States

The Global Structure of the Infla-

Hard Scattering QCD Corrections in

Coset Spaces as Alternatives to Calabi-

Yau Spaces in the Presence of

Electroweak Boson Self-Couplings and

A3 (1988) 1675

A3 (1988) 825

A2 (1987) 1255

A2 (1987) 1263

A2 (1987) 1035

A2 (1987) 1097

A2 (1987) 1025

A1 (1986) 303

A3 (1988) 825

A2 (1987) 739

A3 (1988) 1847

A1 (1986) 211

A2 (1987) 561

A2 (1987) 1359

A2 (1987) 1393

A2 (1987) 453

A2 (1987) 797

A3 (1988) 1751

A3 (1988) 225

A3 (1988) 603

A2 (1987) 1085

A2 (1987) 1097

A2 (1987) 957

The Standard Model and Beyond

Calculation and Phenomenology of

Two Body Decays of Neutralinos \&

A2 (1987) 1145 
R. M. Barnett, M. Drees, D. Karatas, X. Tata and H. Baer

Gunion, J. F., J. L. Hewett, E. Ma and T. G. Rizzo

Gunion, J. F.,

H. E. Haber, M. Drees, X. Tata, R. Godbole, D. Karatas and N. Tracas Gunion, John F. Gurtu, A.

Haber, $\mathrm{H}$.

Haber, H. E.

Haber, H. E.

Haber, H. E.

Haber, Howard E.

Hagiwara, K.

Hagiwara, $\mathrm{K}$.

Halzen, F. and C. S. Kim

Halzen, F.

Hamilton, J. H.

Han, Nguyen Suan

Haupt, T.

Heck, T.

Helayel-Neto, J. A.

Herath-Banda, M. A.

Herrero, $M$.

Herrero, M. J.,

A. Méndez and

T. G. Rizzo

Hewett, J.

Hewett, J. L. and

T. G. Rizzo

Hewett, J. L.

Hewett, J. L. and

S. Pakvasa

Hinchliffe, I.

Hlousek, Zvonimir

Hollowood, T. J. and R. G. Myhill
Charginos To $W, Z$ and Higgs Bosons

Signatures for a New Supersymmet-

A2 (1987) 1199 ric Left-Right Gauge Model from Superstrings

Decays of Higgs Bosons to Neutralinos and Charginos in the Minimal Supersymmetric Model: Calculation and Phenomenology

see Baer, Howard

A2 (1987) 1131

see Banerjee, $\mathrm{S}$.

see Arnowitt, $\mathbf{R}$.

see Gunion, J. F.

see Gunion, J. F.

see Gunion, J. F.

see Baer, Howard

see Arnowitt, R.

see Barger, V.

Associated Production of Weak

Bosons and Heavy Quarks with

Hadron Colliders

see Cudell, J. R.

see Ramayya, A. V.

see Nesterenko, V. V.

see Banerjee, $\mathrm{S}$.

see Ramayya, A. V.

see Smith, A. William

A2 (1987) 1035

A3 (1988) 825

A2 (1987) 1097

A2 (1987) 957

A2 (1987) 1035

A2 (1987) 1145

A2 (1987) 1131

A2 (1987) 1097

A2 (1987) 1181

A2 (1987) 1069

A3 (1988) 1051

A3 (1988) 499

A3 (1988) 2315

A3 (1988) 825

A3 (1988) 499

A1 (1986) 739

A3 (1988) 499

A2 (1987) 1097

see Arnowitt, R.

A2 (1987) 1121

the SSC

see Arnowitt, R.

Heavy Lepton Pair Production From

New $Z$ ' Resonances

see Gunion, J. F.

Hadro-Production of Scalar

Leptoquarks

see Baur, U.

Interbaryonic Force as a Soliton-

Soliton Interaction in Skyrme Model The 112 Breakings of $E_{8}$
A2 (1987) 1097

A2 (1987) 1189

A2 (1987) 1199

A2 (1987) 1299

A2 (1987) 1285

A3 (1988) 2681

A3 (1988) 899 


Holstein, Barry R.
Horvathy, P. A.,
L. O'Raifeartaigh and
J. H. Rawnsley
Hosaka, Atsushi and
Hiroshi Toki
Hou, Bo-Yu, Bo-Yuan
Hou and Pei Wang
Hou, Bo-Yuan
Hou, George W. S.
Hussin, V.
Iengo, R.
Igi, K.
Ikeda, K. and
T. Yamada
Imlay, R.
Itzykson, C.

Jackiw, $\mathbf{R}$.

Jain, Sanjay

Jetzer, P., J. Lacki and L. Mizrachi

Jevicki, A.

Johnson, D. E.

Joshi, P. S. and

J. V. Narlikar

Joshipura, Anjan S.

Kaku, Michio

Kalinowski, M. W.

Kallosh, Renata and Alexei Morozov

Kalyniak P. and M. K. Sundaresan see Donoghue, John F.

A2 (1987) 319

Monopole-Charge Instability

A3 (1988) 665

The Skyrmion and Anti-Skyrmion

A3 (1988) 1413

Chiral Bag Model for the Nucleon

The Static Axially Symmetric Self-Dual

Yang-Mills Fields and Surfaces of

Negative Curvature

see $\mathrm{Hou}, \mathrm{Bo}-\mathrm{Yu}$

A1 (1986) 193

$b \rightarrow s \mu^{+} \mu^{-}$as a Top Mass Meter at the SSC

see Dehin, D.

A3 (1988) 487

see Bonini, M.

A3 (1988) 841

see Arnowitt, R.

A2 (1987) 1097

A3 (1988) 2339

Coupled-Isotriplets Interpretation of

the ${ }_{\Sigma}^{9} \mathrm{Be}$ Hypernuclear State

First Results from the AMY Detector at TRISTAN

Simple Integrable Systems, and Lie Algebras

A2 (1987) 943

A1 (1986) 65

A3 (1988) 285

Connection, Curvature and Phase

Conformally Invariant Field Theory in

Two Dimensions and Strings in

Curved Spacetime

One-Loop Amplitudes in the Heterotic

String

Construction of Interacting String and Superstring Field Theory

Accelerator Physics Issues at the SSC

Conformal Quantization and Space-

Time Singularity

see Govindarajan, T. R.

A3 (1988) 1759

A3 (1988) 243

A3 (1988) 299

A3 (1988) 2503

A1 (1986) 243

A2 (1987) 797

String Field Theory

A2 (1987) 1

Minimal Coupling Scheme for Dirac's

A1 (1986) 227

Field in the Non-Symmetric Theory

of Gravitation

Green-Schwarz Action and Loop

A3 (1988) 1943

Calculations for Superstring

On Mass Shell Renormalization of

One Loop Radiative Corrections in

A3 (1988) 2101 $e^{+} e^{-} \rightarrow W^{+} W^{-}$ 
3022 Cumulative Author Index

Kamran, Mujahid and I. E. Qureshi

Kamran, Mujahid and

I. E. Qureshi

Kang, Kyungsik and Alan R. White

Kapoor, A. K.

Karatas, D.

Karatas, D.

Karatas, D.

Karatas, Debra L.

Karliner, M., I. Klebanov and L. Susskind

Katznelson, E.

Kaul, G. L.

Kawai, H. and

D. C. Lewellen and

S. H. Henry Tye

Ketov, S. V.

Kielanowski, P.

Kim, C. S.

Kim, C. S.

Kim, C. S.

Kim, Y. D.

Kimura, Kazuhiro

Kirillov, A. N. and

F. A. Smirnov

Kirilova, D. P.

Kiritsis, Elias B.

Kiss-Toth, Tibor

Klebanov, I.

Kluger, Y. and

J. M. Eisenberg

Kmiecik, $\mathbf{M}$.

Kobrinsky, M. N.

Kodama, Hideo and

Misao Sasaki

Kodama, Hideo and

Misao Sasaki

Kodama, Hideo
The Chou-Yang Model $\Xi^{-} p \rightarrow \Xi^{-} p$ at

High Energies

The Chou-Yang Model and Elastic $\pi p$

Scattering at High Energies

A Unique SU(5) and SO(10) Unifica-

tion With Complete Dynamical

Symmetry Breaking

see Chaturvedi, S.

see Gunion, J. F.

see Arnowitt, R.

see Gunion, J. F.

see Baer, Howard

Size and Shape of Strings

see Craigie, N. S.

see Banerjee, $S$.

Construction of Four Dimensional

Fermionic String Models with a

Generalized Supercurrent

New $N=2$ Matter Couplings in

Superspace

see Bohm, A.

see Halzen, F.

see Arnowitt, R.

see Cudell, J. R.

see Kwon, O. J.

see Fujitsu, Akira

Form Factors in $\mathrm{O}(3)$ Nonlinear

$\sigma$-Model

see Dolgov, A. D.

Character Formulae and The Structure

of the Representations of the $N=1$,

$N=2$ Superconformal Algebras

Exact Solutions of Nonlinear SU $(N)$

Principal $\sigma$-Models

see Karliner, $M$.

The Isoscalar Charge Form Factor in

Nuclei of $A=2,3$ Obtained in the

Skyrme Model

see Bohm, A.

see Anisovich, V. V.

Evolution of Isocurvature Perturba-

tions I: Photon-Baryon Universe

Evolution of Isocurvature Perturba-

tions II. Radiation-Dust Universe

see Yokoyama, Jun'ichi
A2 (1987) 217

A2 (1987) 1871

A2 (1987) 409

A3 (1988) 163

A2 (1987) 1035

A2 (1987) 1097

A2 (1987) 1145

A2 (1987) 1131

A3 (1988) 1981

A1 (1986) 953

A3 (1988) 825

A3 (1988) 279

A3 (1988) 703

A3 (1988) 1103

A2 (1987) 1069

A2 (1987) 1097

A3 (1988) 1051

A1 (1986) 709

A3 (1988) 2911

A3 (1988) 731

A3 (1988) 267

A3 (1988) 1871

A3 (1988) 1147

A3 (1988) 1981

A3 (1988) 2127

A3 (1988) 1103

A1 (1986) 463

A1 (1986) 265

A2 (1987) 491

A2 (1987) 1809 
Koenig, W.

Komamiya, Sachio

Koonin, S. E.

Krasny, M. W.

Kuhn, D.

Kuo, T.

Kuo, T. K.

Kwon, O. J., Y. D. Kim, Y.S. Myung, B. H. Cho and Y. J. Park

Lacki, J.

Lam, C. S.

Lam, C. S. and S. Y. Lo

Lang, C. B., M. Pilch and B.-S. Skagerstam Leike, A.

Lendvai, E.

Lerda, A., J. E. Nelson and $T$. Regge

Levinas, M. L. Lewellen, D. C.

Lhallabi, T. and

E. H. Saidi

Lhallabi, T. and

E. H. Saidi

Lhallabi, T.

Lichtenberg, D. B.

Linde, A. D.

Lindstrom, U.

Lo, S. Y. and

A. Schreiber

Lo, S. Y. see Ramayya, A. V.

A3 (1988) 499

see Bengtsson, Hans-Uno

A2 (1987) 1055

A3 (1988) 409

A3 (1988) 825

A3 (1988) 825

A2 (1987) 1327

A2 (1987) 1351

A1 (1986) 709

Stability of Schwarzschild Black Hole in the Massive Brans-Dicke Theory

see Jetzer, $P$.

A3 (1988) 243

GSO Projections of Modular-Invariant Aperiodic Strings

Induced Production of Bosons and its Application to Centauro Events

Compactification of Space-Time Dimensions in Lattice Gauge Theory Can the Minimal SUSY Extension of the Standard Model Be Ruled Out by Chargino Production at $e^{+} e^{-}$ Colliders?

see Csikor, F.

Covariant Canonical Formalism for

Polynomial Supergravity in any

Dimensions

see Domenech, $\mathrm{G}$.

see Kawai, $\mathbf{H}$.

The $(4,0)$ and $(4,4)$ Supersymmetric

Nonlinear $\sigma$-Models in the $D=2$

Harmonic Superspace

Extended BRS and Anti-BRS

Symmetries in $N=2$ Harmonic

Superspace

Anomalies of BRS and Anti-BRS

Ward-Identities in $N=1$ Super-

symmetric Theory

Energy Levels of Quarkonia in

Potential Models

see Goncharov, A. S.

First Order Actions for Gravitational

Systems, Strings and Membranes

Partition Temperature and Pion

Condensation in High Energy

Collisions

see Lam, C. S.
A3 (1988) 913

A1 (1986) 451

A3(1988) 1423

A3(1988) 2895

A3 (1988) 2959

A2 (1987) 1643

A3 (1988) 2567

A3 (1988) 279

A3 (1988) 187

A3 (1988) 631

A3 (1988) 647

A2 (1987) 1669

A2 (1987) 561

A3(1988) 2401

A3 (1988) 1603

A1 (1986) 451 
3024 Cumulative Author Index

Loewe, $\mathbf{M}$.

Logunov, A. A. and

Yu. M. Loskutov and

M. A. Mestvirishvili

Lopez, J. L.

Łopuszański, Jan

Łopuszański, Jan

Loskutov, Yu. M.

Lou, Sen-Yue

Lucchesi, Claudio,

Olivier Piguet and

Klaus Sibold

Lykyanov, S. L.

Ma, E.

$\mathrm{Ma}, \mathrm{E}$.

Ma, W. C.

Mackenzie, R. and

Frank Wilczek

Magnea, L.

Magnollay, $\mathrm{P}$.

Mahmood, S.

Maiella, G.

Malhotra, P. K.

Mandl, F.

Mann, Robert B.

Markytan, $\mathbf{M}$.

Marmo, G.

Marrakchi, A. L.

Martin, B.

Marx, George and

Humitaka Sato

Mättig, P.

McKellar, B. H. J.,

M. D. Scadron and

R. C. Warner

McKeon, D. G. C. and

T. N. Sherry

Mebarki, $\mathbf{N}$.

Mendez, A.

Mendez, A.

Mendez, A. see Bohm, A.

A3 (1988) 1103

Relativistic Theory of Gravity

A3 (1988) 2067

see Barger, V.

Locality Properties of Generators of Internal Symmetry with Nonnegative Spectrum

A Remark on B. R. S. Transformations see Logunov, A. A.

see Ni, Guang-Jiong

The Adler-Bardeen Theorem for the

Axial U(1) Anomaly in a General

Non-Abelian Gauge Theory

see Fateev, V. A.

A3 (1988) 507

see Arnowitt, R.

see Gunion, J. F.

see Ramayya, A. V.

Peculiar Spin and Statistics in Two

Space Dimensions

see Del Duca, V.

see Bohm, A.

see Craigie, N. S.

see Cristofano, $\mathrm{G}$.

see Banerjee, $\mathrm{S}$.

see Banerjee, $\mathbf{S}$.

see Sarkar, Utpal

see Banerjee, S.

see Biedenharn, L. C.

see Boutaleb-J, H.

see Ramayya, A. V.

Irreversibility of the Cosmological

Expansion

How Quarks Convert into Jets and

What a Jet Reveals About Quarks-

A Review of $e^{+} e^{-}$Jets

Nonperturbative QCD, The Con-

stituent Quark Model and

Confinement

Operator Regularization and the

Divergence of the Supercurrent

see Contogouris, A. P.

A3 (1988) 2181

A2 (1987) 443

A3 (1988) 2589

A3 (1988) 2067

A3 (1988) 1735

A2 (1987) 385

A2 (1987) 1097

A2 (1987) 1199

A3 (1988) 499

A3 (1988) 2827

A3 (1988) 1081

A3 (1988) 1103

A1 (1986) 953

A2 (1987) 729

A3 (1988) 825

A3 (1988) 825

A3 (1988) 2165

A3 (1988) 825

A2 (1987) 1567

A2 (1987) 179

A3 (1988) 499

A2 (1987) 133

A3 (1988) 1

A3 (1988) 203

see Gunion, J.

see Arnowitt, R.

see Herrero, M. J.
A2 (1987) 785

A2 (1987) 1075

A2 (1987) 1085

A2 (1987) 1097

A2 (1987) 1121 
Mestvirishvili, M. A.

Mijic, Milan B.

Mishra, Amruta

Mishra, H., S. P. Misra

and Amruta Mishra

Misra, S. P.

Mizrachi, L.

Mo, Houjun

Morandi, G.

Moravcsik, Michael J.

Moravcsik, Michael J.

Moravcsik, Michael J.

Morozov, Alexei

Morrison, I.

Mukhanov, V. F.

Mukunda, N.

Müller, Berndt

Müller-Kirsten, H. J. W.

Munoz-Tapia, R.,

J. Taron and

R. Tarrach

Musto, R.

Myhill, R. G.

Myung, Y. S.

Nakagawa, N.

Nandi, S.

Nandi, S.

Nanopoulos, D. V.

Narlikar, J. V.

Nath, $P$.

Nath, Pran

Ne'eman, Yuval and

Djordje Šijački

Nelson, J. E.

Nelson, Philip

Nesterenko, V. A.

Nesterenko, V. V. and.

Nguyen Suan Han

Neufeld, H., W. Grimus

and G. Ecker

Ni, Guang-Jiong, see Logunov, A. A.

A3 (1988) 2067

see Coule, David H.

A3 (1988) 617

A3 (1988) 2331

A3 (1988) 2331

A Variational Approach to the Gross-

Neveu Model

see Mishra, $\mathrm{H}$.

see Jetzer, $\mathbf{P}$.

Probability Functions and Systematics

of Large Scale Clustering

see Biedenharn, L. C.

see Goldstein, Gary R.

see Arash, Firooz

see Goldstein, Gary R.

see Kallosh, Renata

see Wood, L. D.

see Goncharov, A. S.

see Biedenharn, L. C.

see Schäfer, Andreas

see Wiedemann, A.

The Uncertainty of the Gaussian

Effective Potential

see Cristofano, G.

see Hollowood, T. J.

see Kwon, O. J.

see Bagneid, A.

A3 (1988) 2331

A3 (1988) 243

A3 (1988) 1373

A2 (1987) 1567

A1 (1986) 211

A2 (1987) 739

A3 (1988) 1847

A3 (1988) 1943

A3 (1988) 1475

A2 (1987) 561

A2 (1987) 1567

A3 (1988) 1751

A3 (1988) 2349

A3 (1988) 2143

A2 (1987) 729

A3 (1988) 899

A1 (1986) 709

A2 (1987) 1351

A2 (1987) 1097

A2 (1987) 1161

Multilepton Signal From a New

$Z$-Boson at SSC

see Campbell, B. A.

A2 (1987) 831

A1 (1986) 243

A2 (1987) 1097

A2 (1987) 1113

A2 (1987) 1655

$\overline{\mathrm{GL}}(4, R)$ Group-Topology, Covariance

and Curved-Space Spinors

see Lerda, A.

A2 (1987) 1643

A3 (1988) 585

A3 (1988) 1183

A3 (1988) 2315

see Beilin, V. A.

The Hamiltonian Formalism in the

Model of the Relativistic String with

Rigidity

Generalized CP Invariance, Neutral

Flavor Conservation and the Struc-

ture of the Mixing Matrix

The Criterion for Nontrivality of $\lambda \phi^{4}$
A3 (1988) 603

A3 (1988) 1735 
Sen-Yue Lou and Su-Qing Chen

Nicodemi, F.

Nikonov, V. A.

Nikonov, V. A.

Novello, $M$. and

L. A. R. Oliveira

Nyiri, J.

Nyman, Ebbe M. and

D. O. Riska

O'Raifeartaigh, L.

Odintsov, S. D.

Olive, David

Oliveira, L. A. R.

Olness, F.

Olness, F.

Olness, Frederick and

Wu-Ki Tung

Orr, Robert S.

Padmanabhan, $T$.

Padmanabhan, $T$. and

T. R. Seshadri

Padmanabhan, T.,

T. R. Seshadri and

T. P. Singh

Paige, $F$.

Paige, F. E.

Paige, Frank E. and

Edward M. Wang

Paige, Frank E.

Pakvasa, S.

Pakvasa, S.

Pakvasa, Sandip

Pancheri, G. and

Y. Srivastava

Pancholi, S. C.

Park, Y. J.

Pasquinucci, A.

Pati, J. C., Abdus Salam and B. V. Sreekantan

Peris, $\mathbf{S}$.
Model and Its Ultraviolet Stable

Fixed Point

see Cristofano, $\mathrm{G}$.

see Braun, V. M.

see Anisovich, V. V.

A Marionette Universe

see Anisovich, V. V.

Nuclei as Topological Solitons

A2 (1987) 729

A3 (1988) 1301

A1 (1986) 463

A1 (1986) 943

A1 (1986) 463

A3 (1988) 1535

see Horvathy, P. A.

A3 (1988) 665

A3 (1988) 1859

A1 (1986) 303

A1 (1986) 943

A2 (1987) 1025

A2 (1987) 1085

A2 (1987) 1413

A2 (1987) 923

A3 (1988) 1593

A3 (1988) 2113

Quantum Uncertainty in the Horizon

Size in an Inflationary Universe

Uncertainty Principle and the

Quantum Fluctuations of the

Schwarzschild Light Cones

see Arnowitt, R.

see Bengtsson, H.-U.

Standard Model Neutral Higgs

Subgroup Summary

ELMUD: An Electron Muon Detector

for Higgs Physics at the SSC

see Hewett, J. L.

see Barger, V.

Signatures for Horizontal Symmetries and Some Other Exotica at Hadron Colliders

Minimum Bias and Mini-Jets at the SSC

see Ramayya, A. V.

see Kwon, O. J.

see Cecotti, $S$.

Moon as the Searching Ground for

Proton Decay

see Grifols, J. A.
A1 (1986) 491

A2 (1987) 1113

A2 (1987) 1359

A2 (1987) 985

A2 (1987) 993

A2 (1987) 1299

A2 (1987) 1327

A2 (1987) 1317

A2 (1987) 1389

A3 (1988) 499

A1 (1986) 709

A3 (1988) 1675

A1 (1986) 147

A3 (1988) 225 
Perl, Martin L.

Pertermann D. and J. Ranft

Petkova, V. B.

Petrov, A. N. Pettorino, R.

Piekarewicz, J., M. R. Zirnbauer and

S. E. Koonin

Piguet, O. and K. Sibold

Piguet, Olivier

Pilch, $M$.

Plothow-Besch, H.

Pócsik, G.

Popova, A. D. and A. N. Petrov

Poppe, M.

Porrati, M.

Praschifka, J.,

C. D. Roberts and

R. T. Cahill

Putikka, W.

Qureshi, I. E.

Qureshi, I. E.

Rabelo de Carvalho, F. A. B.

Raciti, Mario

Raczka, R.

Radul, A.

Radyushkin, A. V.

Rae, W. D. M.

Rai, B.

Rajpoot, S. and M. A. Samuel see Gan, Kock Kiam

A3 (1988) 531

Study of $Z(N)$ Gauge Theories on a

Three-Dimensional Pseudorandom

Lattice

Two-Dimensional (Half-) Integer Spin

Conformal Theories With Central

Charge $c<1$

see Popova, A. D.

see Cristofano, G.

SU(2) Lattice Gauge Theory in the

Electric-Field Representation

Nonrenormalization Theorems of

Chiral Anomalies and Finiteness

in Supersymmetric Yang-Mills

Theories

see Lucchesi, Claudio

see Lang, C. B.

Latest Results from the UA2

Experiment

see Csikor, F.

Exact Dynamic Theories on a Given

Background in Gravitation

Exclusive Hadron Production in Two-

Photon Reactions

see Cecotti, $S$.

A Study of $\rho \rightarrow \pi \pi$ Decay in a Global

Color Model For QCD

see Barger, V.

see Kamran, Mujahid

see Kamran, Mujahid

see Smith, A. William

see D'Auria, Riccardo

see Barut, A. O.

see Bershadsky, M.

see Beilin, V. A.

Clustering Phenomena and Shell

Effects in Nuclear Structure and

Reactions

see Balachandran, A. P.

Four Preon Composite Quarks and

Leptons
A3 (1988) 2181

A2 (1987) 217

A2 (1987) 1871

A1 (1986) 739

A3 (1988) 1499

A3 (1988) 2945

A3 (1988) 2651

A2 (1987) 729

A3 (1988) 409

A1 (1986) 913

A2 (1987) 385

A3 (1988) 1423

A2 (1987) 899

A3 (1988) 2959

A3 (1988) 2651

A1 (1986) 545

A3 (1988) 1675

A2 (1987) 1797

A3 (1988) 953

A2 (1987) 265

A2 (1987) 165

A3 (1988) 1183

A3 (1988) 1343

A3 (1988) 2621

A2 (1987) 1339 
Ramayya, A. V.,

Levels in ${ }_{38}^{80} \mathrm{Sr},{ }_{39}^{83} \mathrm{Y}$ and ${ }_{40}^{83} \mathrm{Zr}$ and the

M. A. Herath-Banda,

M. Barclay,

J. H. Hamilton,

W. C. Ma,

R. Soundranayagam,

S. C. Pancholi,

H. Yamada,

L. Cleemann, N. Schmal,

J. Eberth, T. Heck,

J. Roth, W. Koenig and

B. Martin

Randjbar-Daemi, S.

Abdus Salam and

J. A. Strathdee

Ranft, J.

Rawnsley, J. H.

Regge, $T$.

Restuccia, A.

Restuccia, A. and

J. G. Taylor

Rindani, Saurabh D.

Ringland, Gordon A.

Riska, D. O.

Ritz, Steven

Riva, Franco

Rizzo, T.

Rizzo, T. G.

Rizzo, T. G.

Rizzo, T. G.

Roberts, C. D.

Rodriguez-Trias, R.

Ross, R. T.

Roth, J.

Rubakov, V. A.

Saidi, E. H.

Saidi, E. H.

Saidi, E. H.

Saidi, E. H.

Salam, Abdus
On the Hypermultiplet Theories

see Pati, J. C.
New Region of Strong Deformation around $N=Z \approx 38$

$\sigma$-Models and String Theories

A2 (1987) 667

A3 (1988) 1499

A3 (1988) 665

A2 (1987) 1643

A3 (1988) 451

A3 (1988) 2855

see Bressloff, P. C.

Constructing the Superstring Space-

Time SUSY Algebra in the Light-

Cone Gauge

see Govindarajan, T. R.

A2 (1987) 797

see Goldstein, Gary R.

see Nyman, Ebbe M.

Search for Neutral Higgs at LEP 200

A3 (1988) 1847

A3 (1988) 1535

A2 (1987) 1013

A3 (1988) 953

A2 (1987) 1097

A2 (1987) 1121

A2 (1987) 1189

A2 (1987) 1199

A2 (1987) 1797

A3 (1988) 943

A3 (1988) 825

A3 (1988) 499

A3 (1988) 1199

see Deryagin, D. V.

Renormalizable $N=2$ Super-

A2 (1987) 695

symmetric and Gauge Invariant

Interactions from the $N=2$

Harmonic Superspace with Central

Charges

see Lhallabi, $\mathbf{T}$.

A3 (1988) 187

A3 (1988) 631

A3 (1988) 861

A1 (1986) 147 
Salam, Abdus

Saleem, Mohammad

Samuel, J.

Samuel, M. A.

Sanchez, N.

Sánchez, N.

Santos, A. Lima

Sarkar, Utpal

Sarkar, Utpal and

Robert B. Mann

Sarker, A. K.

Sartori, G.

Sasaki, Misao

Sasaki, Misao

Sato, Humitaka

Sato, Katsuhiko

Sato, Nobuaki

Sazdjian, H.

Scadron, M. D.

Schäfer, Andreas,

Berndt Müller and

Walter Greiner

Schaposnik, F. A. and

H. Vucetich

Schmal, N.

Schmitz, Norbert

Schreiber, A.

Schwarz, A. S.

Schwarz, John H.

Serdaroglu, Meral

Serebryakov, M. S.

Seshadri, T. R.

Seshadri, T. R.

Sevrin, A.

Shabelski, Yu. M. see Randjbar-Daemi, S.

A2 (1987) 667

The Chou-Yang Model and $\pi^{-} p$

Elastic Scattering

see Biedenharn, L. C.

see Rajpoot, S.

Generalized Analytic Mappings and

Flat-Space Models of Hawking

Radiation

see Domenech, G.

see Abdalla, E.

see Govindarajan, T. R.

Neutrino Masses in Superstring

Theories with Intermediate Scales

see Banerjee, S. N.

see Gatto, R.

see Kodama, Hideo

see Kodama, Hideo

see Marx, George

see Yokoyama, Jun'ichi

see Yokoyama, Jun'ichi

The Massless Bound State Formalism

in Two-Particle Relativistic Quantum

Mechanics

see McKellar, B. H. J.

Local Vacuum Excitations in Strong

Electromagnetic Fields?

Quantization of Gravitation with Weyl

Fermions

see Ramayya, A. V.

Hadron Production in High Energy

Lepton-Nucleon and Lepton-Nucleus

Collisions

see Lo, S. Y.

see Baranov, M. A.

Review of Recent Developments in

Superstring Theory

$E_{6}$ Phenomenology, A $3 \times 3$ Matrix

Approach

Radiative Corrections to the $S$-Wave

Dirac Equation Near a Monopole and

Monopole Catalysis of Proton Decay

see Padmanabhan, $T$.

see Padmanabhan, $T$.

see Gastmans, R.

see Braun, V. M.
A2 (1987) 1567

A2 (1987) 1339

A3 (1988) 1123

A3 (1988) 2567

A2 (1987) 753

A2 (1987) 797

A3 (1988) 2165

A2 (1987) 1829

A1 (1986) 683

A1 (1986) 265

A2 (1987) 491

A2 (1987) 133

A2 (1987) 1809

A2 (1987) 1809

A3 (1988) 1235

A3 (1988) 203

A3 (1988) 1751

A2 (1987) 1755

A3 (1988) 499

A3 (1988) 1997

A3 (1988) 1603

A2 (1987) 1773

A2 (1987) 593

A2 (1987) 1617

A2 (1987) 1887

A1 (1986) 491

A3 (1988) 2113

A2 (1987) 195

A3 (1988) 2417
A2 (1987) 485 
3030 Cumulative Author Index

Sharatchandra, H. S.

Sherry, T. N.

Shifman, M. A.

Shih, C. C.

Shirkov, D. V.

Shono, Y.

Shore, G. M. and

G. Veneziano

Sibold, K.

Sibold, Klaus

Siegel, W.

Siegel, W.

Šjački, Djordje

Singh, $T$. and

Tarkeshwar Singh

Singh, T. P.

Singh, T. P. and

T. Padmanabhan

Singh, Tarkeshwar

Sjostrand, T.

Skagerstam, B.-S.

Skyrme, T. H. R.

Smirnov, F. A.

Smith, A. William,

F. A. B. Rabelo de

Carvalho and

J. A. Helayel-Neto

Solà, J.

Soni, A.

Soni, A.

Sorba, P. and

B. Torresani

Soundranayagam, $\mathbf{R}$.

Sparano, G.

Squarcia, S.

Sreekantan, B. V.

Srinivasan, V.

Srivastava, A. M.

Srivastava, Y.

Stanev, Yassen S. and

Ivan $\mathrm{T}$. Todorov

Strathdee, J. see Anishetty, R.

A3 (1988) 875

see McKeon, D. G. C.

A2 (1987) 785

Theory of Weak Interactions: Recent

Development and Problems

see Carruthers, $\mathbf{P}$.

Renormalization Group in Modern

Physics

see Bandō, $\mathbf{H}$.

Current Algebra and Supersymmetry

see Piguet, 0 .

see Lucchesi, Claudio

First- and Second-Class Constraints

in Super-BRST

Conformal Invariance of Extended

Spinning Particle Mechanics

see Ne'eman, Yuval

General Class of Scalar-Tensor

Theories-A Review

see Padmanabhan, $T$.

An Attempt to Explain the Smallness

of the Cosmological Constant

see Singh, $T$.

Status of Fragmentation Models

see Lang, C. B.

The Origins of Skyrmions

see Kirillov, A. N.

On the Auxiliary Fields of Supergravity

Theories

see Grifols, J. A.

see Cox, B.

see Bengtsson, H.-U.

Twisted Vertex Operator and String

Theory

see Ramayya, A. V.

see Balachandran, A. P.

see Banerjee, S.

see Pati, J. C.

see Chaturvedi, $\mathbf{S}$.

see Balachandran, A. P.

see Pancheri, G.

Towards a Conformal QED $_{4}$ With a

Nonvanishing Current 2-Point

Function

Kaluza-Klein Theory
A3 (1988) 225

A3 (1988) 2769

A2 (1987) 1447

A3 (1988) 1321

A3 (1988) 1581

A1 (1986) 499

A1 (1986) 913

A2 (1987) 385

A3 (1988) 2707

A3 (1988) 2713

A2 (1987) 1655

A2 (1987) 645

A1 (1986) 491

A3 (1988) 1593

A2 (1987) 645

A3 (1988) 751

A3 (1988) 1423

A3 (1988) 2745

A3 (1988) 731

A1 (1986) 739

A2 (1987) 1221

A2 (1987) 1241

A3 (1988) 1451

A3 (1988) 499

A3 (1988) 2621

A3 (1988) 825

A1 (1986) 147

A3 (1988) 163

A3 (1988) 2621

A2 (1987) 1389

A3 (1988) 1023

A1 (1986) 1 
Strathdee, J.

Strathdee, J. A.

Sudarshan, E. C. G.

Sudhakar, K.

Sugano, K.

Sugimoto, Shojiro

Sundaresan, M. K.

Susskind, L.

Suzuki, Mahiko

Svozil, Karl and

Anton Zeilinger

Szczekowski, M.

Takaki, $\mathrm{H}$.

Tanii, Yoshiaki and

Yoshiyuki Watabiki

Tao, Charling

Taron, J.

Tarrach, $R$.

Tata, X.

Tata, $X$

Tata, X.

Tata, Xerxes R.

Taylor, J. G.

Taylor, J. G.

Tchrakian, D. H.

Tesima, Kiusau

Thompson, G.

Todorov, Ivan $\mathrm{T}$.

Toki, Hiroshi

Tonin, $\mathbf{M}$.

Torresani, B.

Tracas, N.

Tracas, $\mathbf{N}$.

Treleani, D.

Troost, W.

Tseytlin, A. A.

Tu, Tung-sheng

Tung, $\mathrm{Wu}-\mathrm{Ki}$

Tung, Wu-Ki

Twisk, $\mathrm{S}$.
Extended Poincare Supersymmetry

see Randjbar-Daemi, S.

see Biedenharn, L. C.

see Banerjee, $S$.

Properties of Gluon Jets: A Review

Present Status of VENUS

see Kalyniak, P.

see Karliner, $M$.

Composite Weak Bosons at Supercolliders

Dimension of Space-Time

see Banerjee, $\mathrm{S}$.

see Bandō, $\mathrm{H}$.

Vertex Functions in the Path Integral

Formalism of Open String Theories

The UA1 Experiment

see Munoz-Tapia, $R$.

see Munoz-Tapia, R.

see Gunion, J. F.

see Arnowitt, R.

see Gunion, J. F.

see Baer, Howard

see Bressloff, P. C.

see Restuccia, $A$.

see Wiedemann, A.

The Angular Ordering in Soft-Gluon Emission

see Delbourgo, $\mathbf{R}$.

see Stanev, Yassen S.

see Hosaka, Atsushi

Superstrings, $k$-Symmetry and Superspace Constraints

see Sorba, P.

see Gunion, J. F.

see Arnowitt, $\mathbf{R}$.

see Ametller, Ll.

see Gastmans, R.

String Theory Effective Action: String

Loop Corrections

see $\mathrm{Yu}$, Hongwei

Parton Distribution Functions and the

QCD-Improved Parton Model-An

Overview

see Olness, Frederick

see Delbourgo, R.
A2 (1987) 273

A2 (1987) 667

A2 (1987) 1567

A3 (1988) 825

A3 (1988) 2249

A2 (1987) 911

A3 (1988) 2101

A3 (1988) 1981

A2 (1987) 1333

A1 (1986) 971

A3 (1988) 825

A3 (1988) 1581

A3 (1988) 2601

A1 (1986) 749

A3 (1988) 2143

A3 (1988) 2143

A2 (1987) 1035

A2 (1987) 1097

A2 (1987) 1145

A2 (1987) 1131

A3 (1988) 451

A3 (1988) 2855

A3 (1988) 2349

A2 (1987) 1425

A3 (1988) 435

A3 (1988) 1023

A3 (1988) 1413

A3 (1988) 1519

A3 (1988) 1451

A2 (1987) 1035

A2 (1987) 1097

A3 (1988) 521

A2 (1987) 195

A3 (1988) 365

A1 (1986) 481

A2 (1987) 1369

A2 (1987) 1413

A3 (1988) 435 
3032 Cumulative Author Index

Tye, S. H. Henry

Tze, Chia-Hsiung

Valencia, German

van Dam, $\mathrm{H}$.

van Nieuwenhuizen, $\mathbf{P}$.

van Nieuwenhuizen, $P$.

Van Proeyen, A.

Vasiliev, M. A.

Veneziano, G.

Veneziano, G.

Villasante, $M$.

Viswanathan, K. S. and

Zhou Xiaoan

Vucetich, $\mathrm{H}$.

Wang, Edward $M$.

Wang, Edward M.

Wang, Mian

Wang, Pei

Warner, R. C.

Watabiki, Yoshiyuki

Weinberg, Steven

Weinberg, Steven

Weiskop, Shlomo

Wheeler, J. A.

Whisnant, K.

Whisnant, $\mathrm{K}$.

Whisnant, K.

Whisnant, $\mathrm{K}$.

Whisnant, $\mathrm{K}$.

White, Alan R.

White, Alan R.

Wiedemann, A.,

H. J. W. Müller-

Kirsten and

D. H. Tchrakian

Wilczek, Frank see Kawai, $\mathbf{H}$.

Manifold-Splitting Regularization,

Self-Linking, Twisting, Writhing

Numbers of Space-Time Ribbons and

Polyakov's Proof of Fermi-Bose

Transmutations

see Donoghue, John F.

see Biedenharn, L. C.

Spinning Strings as Conformal

Supergravities

see Del Duca, V.

see Gastmans, R.

see Fradkin, E. S.

see Shore, G. M.

see Amati, D.

see Cecotti, S.

Free Energy and the Static Potential for Open Smooth Strings

see Schaposnik, F. A.

see Paige, Frank E.

Is the Heavy Higgs Boson Observable at the SSC?

see $\mathrm{Yu}$, Hongwei

see $\mathrm{Hou}, \mathrm{Bo}-\mathrm{Yu}$

see McKellar, B. H. J.

see Tanii, Yoshiaki

Particle Physics: Past and Future

Opportunities for Particle Physics:

Solar Neutrinos and Superstrings

Effects of Compositeness in Top Decay

Geometrodynamic Steering Principle

Reveals the Determiners of Inertia

see Arnowitt, R.

see Barger, $V$.

see Barger, $V$.

see Barger, $V$.

see Barger, $\mathrm{V}$.

see Braaten, Eric

see Kang, Kyungsik

Investigation of a Theory With Soliton-

Like Configurations

see Mackenzie, $\mathbf{R}$.
A2 (1987) 319

A2 (1987) 1567

A1 (1986) 155

A3 (1988) 279

A3 (1988) 1959

A3 (1988) 1081

A2 (1987) 195

A3 (1988) 2983

A1 (1986) 499

A3 (1988) 1615

A2 (1987) 1839

A3 (1988) 2195

A2 (1987) 1755

A2 (1987) 985

A2 (1987) 1001

A1 (1986) 481

A1 (1986) 193

A3 (1988) 203

A3 (1988) 2601

A1 (1986) 135

A2 (1987) 301

A3 (1988) 2929

A3 (1988) 2207

A2 (1987) 1097

A2 (1987) 1171

A2 (1987) 1327

A3 (1988) 879

A3 (1988) 1907

A1 (1986) 693

A2 (1987) 409

A3 (1988) 2349

A3 (1988) 2827 


Willcox, Charles R.
Witten, Edward
Wood, L. D. and
I. Morrison
Wu, Weimin
Wu, Z. C.
Yamada, H.
Yamada, T.
Yamagishi, Hidenaga
Yamamoto, Hiroaki
Yang, Chen Ning
Yang, Chen Ning
Yau, Shing-Tung
Ye, Minghan and
Zhipeng Zheng
Yokosawa, Akihiko

Yokoyama, Jun'ichi, Hideo Kodama, Katsuhiko Sato and Nobuaki Sato

Yu, Hongwei, Wang Mian and Tu Tung-sheng Yu Grigoriev, D. Zamolodchikov, A. B.

Zandron, $\mathrm{O}$.

Zandron, $\mathrm{O}$.

Zeilinger, Anton

Zeppenfeld, D.

Zeppenfeld, D.

Zeppenfeld, Dieter

Zheng, Zhipeng, Zhou, Xiaoan Zimerman, A. H. Zirnbauer, M. R. Zwirner, Fabio see Braaten, Eric

Topological Tools in 10-Dimensional Physics

A Bose-Fermi Map in the SU(3) Limit of the IBA

The ALEPH Experiment at LEP see Fang, L. Z.

see Ramayya, A. V.

see Ikeda, $K$.

Invariant Correlation Functions, Superconvergence Sum Rules, and Electric-Magnetic Duality see Bengtsson, Hans-Uno see Chou, T. T. see Chou, T. T.

A Survey on the Interaction Between Mathematical Physics and Geometry BEPC, The Beijing Electron-Positron Collider

Polarized-Proton and Antiproton Beams at Fermilab and Associated Experiments

Baryogenesis in the Inflationary Universe-The Instantaneous Reheating Model

Discrete Symmetries for Stabilizing the Proton in SU(N) GUTs and the Corresponding Phenomenology see Deryagin, D. V.

Integrals of Motion in Scaling 3-State Potts Model Field Theory see Abecasis, $\mathrm{C}$.

see Foussats, A.

see Svozil, Karl

see Baur, U.

Diagonalization of Color Factors

Substructure and Horizontal Symmetries at Hadron Colliders (Summary Report)

see Minghan, Ye see Viswanathan, K. S.

see Aratyn, $\mathbf{H}$.

see Piekarewicz, J.

Phenomenological Aspects of $E_{6}$ Superstring-Inspired Models
A1 (1986) 693

A1 (1986) 39

A3 (1988) 1475

A1 (1986) 117

A1 (1986) 887

A3 (1988) 499

A3 (1988) 2339

A3 (1988) 1155

A2 (1987) 1055

A1 (1986) 415

A2 (1987) 1727

A1 (1986) 881

A2 (1987) 1707

A3 (1988) 2753

A2 (1987) 1809

A1 (1986) 481

A3 (1988) 1199

A3 (1988) 743

A3 (1988) 2303

A3 (1988) 2971

A1 (1986) 971

A2 (1987) 1285

A3 (1988) 2175

A2 (1987) 1273

A2 (1987) 1707

A3 (1988) 2195

A1 (1986) 421

A3 (1988) 409

A3 (1988) 49 